\title{
Using direct nanoimprinting of ferroelectric films to prepare devices exhibiting bi-directionally tunable surface plasmon resonances
}

\author{
H L Chen ${ }^{1,4}$, K C Hsieh ${ }^{1}$, C H Lin ${ }^{2}$ and S H Chen ${ }^{3}$ \\ ${ }^{1}$ Department of Materials Science and Engineering, National Taiwan University, \\ Taipei 10617, Taiwan, Republic of China \\ ${ }^{2}$ Institute of Electro-Optical Science and Engineering, National Cheng Kung University, \\ Tainan 70101, Taiwan, Republic of China \\ ${ }^{3}$ National Nano Device Laboratory, Hsinchu 30078, Taiwan, Republic of China \\ E-mail: hsuenlichen@ntu.edu.tw
}

Received 28 May 2008, in final form 14 August 2008

Published 22 September 2008

Online at stacks.iop.org/Nano/19/435304

\begin{abstract}
In this paper, we describe an imprint method for the fabrication of bi-directionally tunable surface plasmon resonance (SPR) filters. A periodic metal/ferroelectric film stack exhibiting SPR phenomena was directly imprinted using a sharp mold without the need for a polymer-based resist. Both the refractive index of the surrounding lead zirconate titanate (PZT) films and the period of the textured PZT/metal/PZT structure were dependent upon both the absolute value and sign of the applied potential. The SPR wavelength of the $\mathrm{PZT} /$ gold/PZT-based tunable filter varied over a range of greater than $100 \mathrm{~nm}$ when applying potentials ranging from 0 to $-15 \mathrm{~V}$. This imprinting method has great potential for use in the fabrication of tunable optical filters without the need for complicated processes or specific materials.
\end{abstract}

(Some figures in this article are in colour only in the electronic version)

\section{Introduction}

Surface plasmon resonance (SPR) is the coherent fluctuation of electron density on the surface of a material displaying metallic behavior at the interface with a dielectric medium [1, 2]. According to Ebbesen et al, the transmission peak of the extraordinary optical transmission of sub-wavelength metal hole arrays corresponds to the resonant frequency of the surface plasmon polaritons (SPPs) [3, 4], i.e. the enhanced transmission can be attributed to the resonant interaction of the incident light with the SPPs. In addition, they reported that continuous metallic films possessing appropriate corrugation could also exhibit enhanced transmission [4]. When the metal film is sufficiently thin, the surface plasmon modes of the two

\footnotetext{
4 Address for correspondence: Department of Materials Science and Engineering, National Taiwan University, No. 1, Sec. 4, Roosevelt Road, Taipei 10617, Taiwan, Republic of China
}

surfaces can overlap and interact on both sides of the patterned metal film. Therefore, extraordinary transmission phenomena are also found in continuously corrugated metal films that do not feature perforated hole arrays.

SPR contributes to various novel processes in nanooptics and to localized field enhancement in near-field measurements [5-7]. By introducing apertures or grooves into metallic films to induce the formation of SPPs, the transmission of light can be improved dramatically [3, 7]. For a metallic array of period $P$, the location of $\lambda_{\max }$ in the normal incidence transmittance spectra can be identified approximately from the following dispersion relationship [2]:

$$
\lambda_{\max }=\frac{P}{\sqrt{i^{2}+j^{2}}} \cdot \sqrt{\frac{\varepsilon_{\mathrm{m}} \cdot \varepsilon_{\mathrm{d}}}{\varepsilon_{\mathrm{m}}+\varepsilon_{\mathrm{d}}}}
$$

where the indices $i$ and $j$ are scattering orders from the periodic structure, $\varepsilon_{\mathrm{m}}$ is the real part of the metal's dielectric 
constant and $\varepsilon_{\mathrm{d}}$ is the dielectric constant of the neighboring dielectric material. From equation (1), we deduce that varying the period or dielectric constant of the surrounding medium allows the SPR wavelength to be modulated.

Nanoimprinting lithography (NIL), a potential candidate for next-generation lithographic technology, allows rapid, large-area and low-cost patterning of polymer-based resists. The standard NIL technique uses a thermoplastic resist and defines patterns through physical deformation of deformable polymer materials (resists) by heating them above their glass transition temperature $\left(T_{\mathrm{g}}\right)[8,9]$. After removing the mold, the pattern is transferred to the underlying substrates through etching processes. Direct patterning of the underlying materials-i.e. without etching-would accelerate the fabrication processes. Such an approach has been employed in the embossing of silicon substrates through laserassisted direct imprinting (LADI) [10], which used a highpower excimer laser $\left(\mathrm{XeCl}, 1.6 \mathrm{~J} \mathrm{~cm}^{-2}\right)$ to melt silicon while simultaneously applying external pressure. This approach has limited applicability because not all materials can tolerate such high-intensity irradiation. Recently, a process was reported for the direct imprinting of metal films under ultra-high pressure (several hundred megapascals) within an oil press imprint instrument. Again, this process has limited applicability because it might damage underlying substrates or devices. Furthermore, because ceramic films, such as ferroelectric films, are harder than metal films, much higher pressures might have to be applied in such a direct imprinting process [11].

Ferroelectric films are of interest for their application to sensors, actuators, non-volatile memories and optoelectronic devices because they exhibit piezoelectric and ferroelectric properties $[12,13]$. The patterning of various profiles of ferroelectric films with sub-micrometer features is important for the fabrication of microelectromechanical and optoelectronic systems and optoelectronic applications $[14,15]$. Nevertheless, many kinds of ferroelectric films, such as lead zirconate titanate (PZT), are not easy to pattern using conventional lithography and etching processes. In a previous study, we successfully patterned bi-layers of metal/ferroelectric films through direct NIL, using a sharp mold and a soft gel film to imprint ferroelectric films directly at low pressure $(\leqslant 20 \mathrm{MPa})$ and temperature [16].

The nature of the dielectric constant of a liquid coming into contact with an array of metallic holes has an effect on its SPR phenomena [17]. In addition, the refractive index of a liquid crystal medium contacting a periodic metal surface was modulated by applying an external voltage, thereby allowing selective control over the wavelength of the transmitted light; in this case, the periodic metal surface was first fabricated using complicated lithographic processes and then filled with the liquid crystal. Applying an external potential of $30 \mathrm{~V}$ to the surrounding liquid crystal allowed the SPR wavelength to be varied over a range of $20 \mathrm{~nm}$ [18].

Ferroelectric materials have large dielectric constants and refractive indices, which can strongly influence the local fields experienced around them. The unique phenomena exhibited by such ferroelectric materials as PZT allow the fabrication of high-quality polycrystalline films exhibiting enhanced electric and ferroelectric properties relative to those of bulk ceramics. Furthermore, PZT films can exhibit a sufficient change in refractive index when an electric field is applied, making them suitable for application in electro-optical and piezoelectric devices [19]. In this study, we used the direct imprinting process to fabricate tunable filters based on SPR phenomena. We surrounded the periodic metal surfaces with PZT films, patterning the corrugated metal and PZT stack in one imprinting step. Combining the piezoelectric properties of PZT films with the SPR phenomena of periodic metal structures, we studied the tunability of the latter when applying external electric fields. Furthermore, the corrugated PZT/metal/PZT stack could also be compressed or extended when the external electric fields were applied in certain directions. Thus, the period of the corrugated metal/PZT structures could also be tuned by applying an external electric field, which also affected the properties of the surface plasmon of the periodic metal structures. When we applied an electric field to the PZT/metal/PZT structure, the refractive index of the surrounding PZT films and the period of the metal structure both changed as a result of the piezoelectric properties of the PZT films. Therefore, we believe that our technique for fabricating metal/ferroelectric structures exhibiting variable tuning phenomena will make surface plasmon-based filters more applicable for use in future devices.

\section{Experiment}

Ferroelectric PZT films were patterned (figure 1) by imprinting gel films, which were prepared by metal-organic decomposition (MOD) of solutions (Kojundo, Japan). The PZT gel films were spin-coated onto glass and $\mathrm{SrTiO}_{3}$ substrates at a thickness of approx. $200 \mathrm{~nm}$. A gold film having a thickness of approx. $50 \mathrm{~nm}$ was then deposited on the PZT film to form a gold/PZT stack. The silicon molds used in these experiments were fabricated through sequential electron beam lithography (Leica, Weprint-200) and reactive ion etching. A high-density-plasma reactiveion-etching (HDP-RIE) system (Duratek, Mutiplex Cluster) equipped with an inductively coupled plasma (ICP) source was used to fabricate the sharp molds [20]. During the imprinting processes, an imprinting pressure of approx. $20 \mathrm{MPa}$ was applied at room temperature. After imprinting, the PZT film was coated on the patterned gold/PZT stack to form a PZT/Au/PZT structure. The samples were first dried at $120^{\circ} \mathrm{C}$ for $30 \mathrm{~min}$ and then pyrolyzed at $450{ }^{\circ} \mathrm{C}$ for $30 \mathrm{~min}$ in air. Finally, the samples were annealed at $650-800^{\circ} \mathrm{C}$ for $60 \mathrm{~min}$ to obtain the perovskite phase. The surface profiles of the patterned films were observed using a JEOL JSM6500F scanning electron microscope (SEM). Optical spectra were measured using a Hitachi U4100 spectrophotometer. The ellipsometric parameters $\Psi$ and $\Delta$ of the PZT films were measured using a Sopra SE5 ellipsometer. The ellipsometric data were fitted using a classical Lorentz oscillator model for the acquisition of refractive indices. The piezoelectric properties of the PZT films under various external applied potentials were measured using a Seiko SPA-300 electrostatic force microscope. 


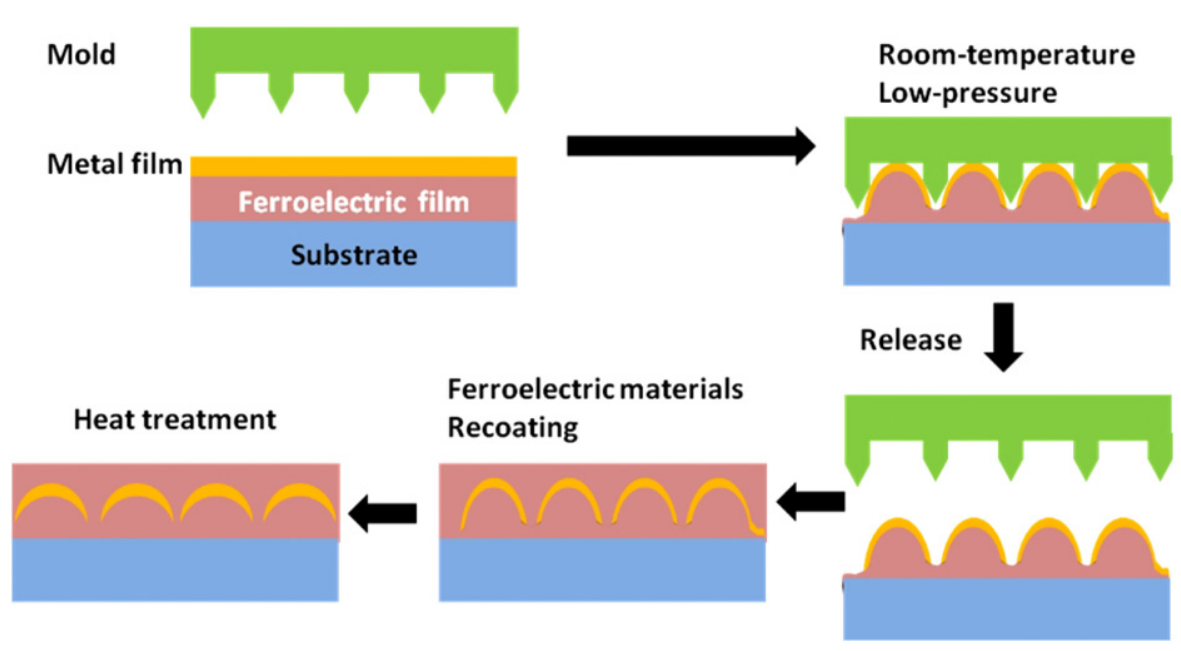

Figure 1. Schematic representations of the process for nanoimprinting for the patterning patterned metal/ferroelectric structures.
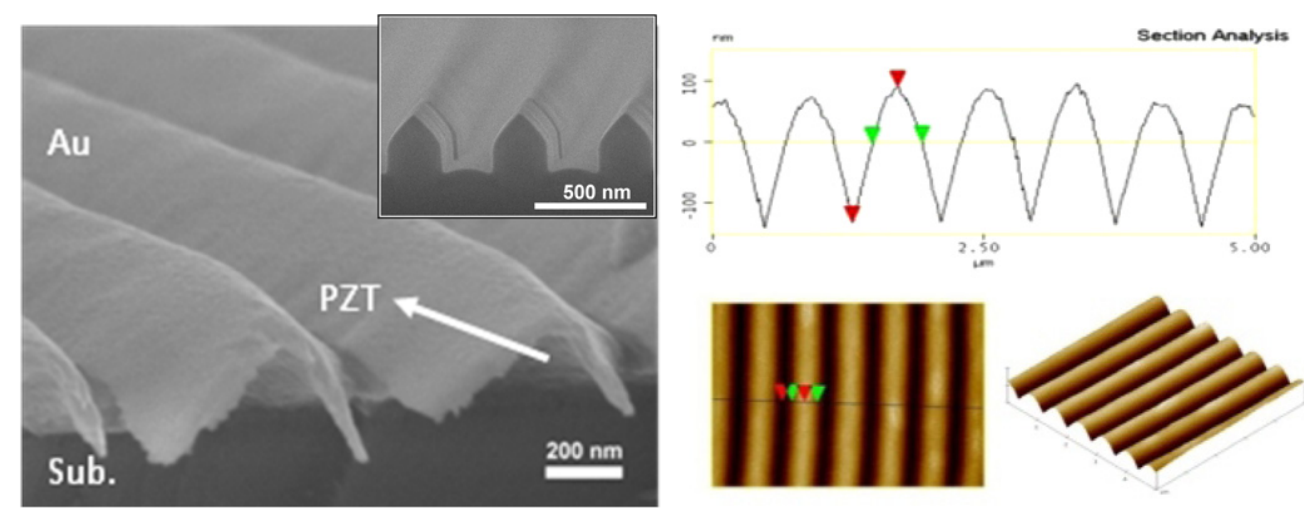

(a)

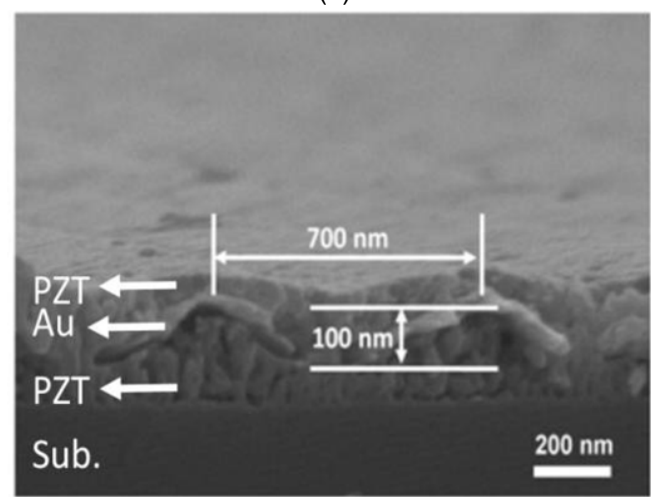

(b)

Figure 2. (a) SEM and AFM images of the mold and the patterned gold/PZT structure. (b) SEM image of the PZT/gold/PZT structure after sintering process.

\section{Results and discussion}

Figure 2 displays microscopy images of directly nanoimprinted patterned ferroelectric films. The inset of figure 2(a) displays an SEM image of a sharp silicon mold used to imprint the gold/PZT structure. Figure 2(a) also displays SEM and atomic force microscopy (AFM) images of patterned gold/PZT structures. The depth and period of the gold/PZT structure were approx. 200 and $800 \mathrm{~nm}$, respectively. To improve their piezoelectric properties, it was necessary to sinter the patterned PZT films at high temperature, but this process led to serious shrinking and destruction of the patterns. To avoid this problem, we placed a PZT cover film as a coat over the bilayer gold/PZT structure, forming a PZT/gold/PZT sandwich structure. This sandwich structure prevented serious distortion of the patterned gold/PZT structure by balancing the shear stresses experienced during treatment at high temperature. 


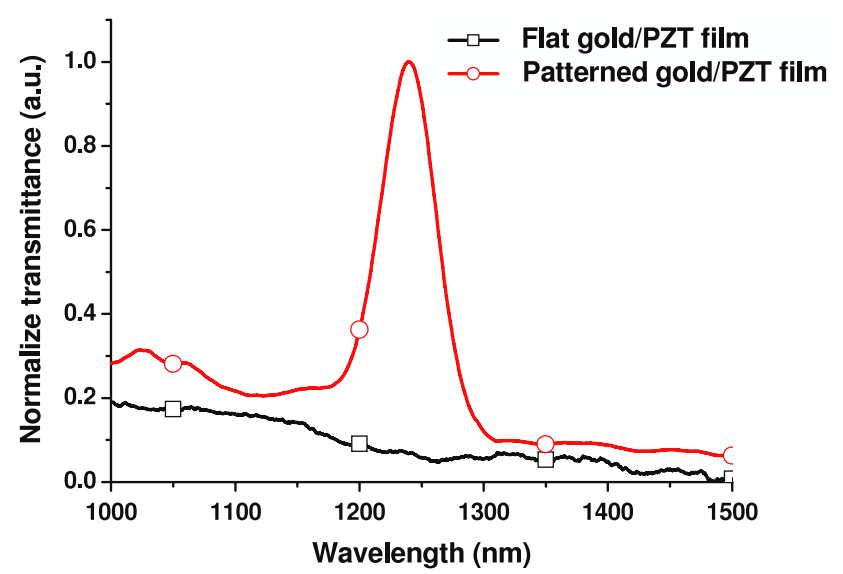

Figure 3. Transmission spectra of gold/PZT films before and after patterning with periodic structures.

Figure 2(b) indicates that the period of the PZT/gold/PZT structure reduced to approx. $700 \mathrm{~nm}$ after sintering.

To study the electro-optical properties of ferroelectricbased SPR filters, we fabricated the patterned PZT/gold/PZT structure on a $\mathrm{SrTiO}_{3}$ bottom electrode and covered it with an indium tin oxide (ITO) glass as the top electrode. Applying an external electric field to this patterned PZT/gold/PZT structure provided a tunable SPR filter.

To study the extraordinary transmission phenomena, we used an optical spectrometer to measure the transmission spectra of the textured gold/PZT structure (figure 3). For a flat gold film having a thickness of $50 \mathrm{~nm}$, we observe a small degree of transmission that varied only with respect to the thickness of the gold film. After performing the imprinting process, however, the transmission spectrum of the corrugated gold/PZT structure displayed an obvious transmission peak at $1240 \mathrm{~nm}$ that arose from the SPR effect.

Based on this patterned gold/PZT structure, we applied an external electric field in an attempt to change either the refractive index of the surrounding PZT film or the period of the patterned PZT/gold/PZT structure to tune the SPR wavelength. Figure 4 provides a graphical representation of an ITO glass-covered patterned PZT/gold/PZT sample subjected to positive and negative applied electric fields; figure 5 displays the corresponding optical spectra. In figure 5(a), the SPR wavelength of a patterned sandwich structure appeared at approx. $1169 \mathrm{~nm}$ in the initial state (no applied potential). Upon applying a potential of $+5 \mathrm{~V}$, the SPR peak blueshifted to $1148 \mathrm{~nm}$. Increasing this potential to $+7 \mathrm{~V}$ caused the SPR peak to shift in the opposite direction, i.e. to a longer wavelength of approx. $1158 \mathrm{~nm}$. Further increases to +10 and $+15 \mathrm{~V}$ led to dramatic redshifts of the SPR peak to 1227 and $1244 \mathrm{~nm}$, respectively.

Next we prepared a second patterned PZT/gold/PZT sample to study the effects of negative applied electric fields. The SPR wavelength appeared at approx. $1135 \mathrm{~nm}$ for this patterned sandwich structure in the absence of an applied potential (figure 5(b)). After applying potentials of $-5,-7$, -10 and $-15 \mathrm{~V}$, the SPR peak redshifted continuously to 1151 , 1164, 1246 and $1256 \mathrm{~nm}$, respectively — a total wavelength shift of over $120 \mathrm{~nm}$.

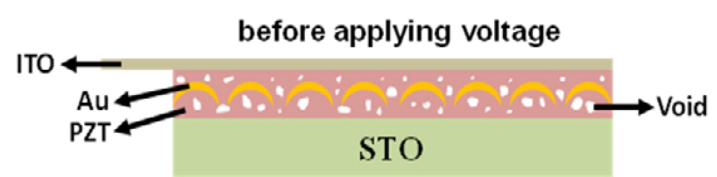

(a)

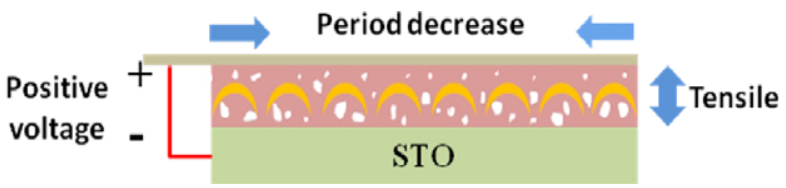

(b)

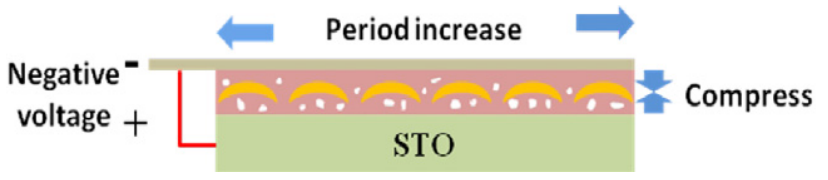

(c)

Figure 4. Schematic cartoon diagram representations of patterned PZT/gold/PZT structures (a) before in the absence of an applying applied voltage potential and ((b), (c)) after, (b) applying positive voltage and (c) applying negative voltage potentials, respectively.

The SPR wavelengths of periodic metallic structures are highly sensitive to their periods and to the refractive index of their surrounding materials. We suspected that the tunable SPR phenomena exhibited by our devices were influenced by both of these factors. A redshift in the SPR band is induced by either increasing the period of the metallic structure or increasing the refractive index of its surrounding medium; correspondingly, a blueshift is induced by decreasing the period or decreasing the refractive index. First, let us consider the refractive index change of the PZT films. We used an ellipsometer to measure the refractive indices of PZT films before and after applying an external potential. The measured refractive index of the PZT film was 2.303 in the absence of an applied voltage. After applying both positive and negative external potentials of 5,10 and $15 \mathrm{~V}$, we measured the refractive indices of PZT films to be 2.310, 2.397 and 2.410, respectively. Thus, the refractive indices of PZT films increased upon increasing the amount of positive or negative potential. For refractive index increments $\left(\Delta n_{\mathrm{PZT}}\right)$ of $0.007,0.094$ and 0.107 , the SPR wavelength shifts calculated from equation (1) should be 5, 80 and $90 \mathrm{~nm}$, respectively. Because these calculated values do not match the measured values from figure 5, the refractive index changes of the PZT films were not solely responsible for the SPR phenomena.

The patterned PZT/gold/PZT structures possessed voids (figure 4(a)) within their PZT films prior to applying a potential. Figure 2(b) suggests that the voids might have formed as a result of constriction of the PZT films during the sintering process, which would affect the effective refractive index of the surrounding PZT films and have an uncertain effect on the SPR wavelength shift. As displayed in figure 4(b), the patterned PZT/gold/PZT structure was subjected to tension under applied positive voltages. We propose that the period of the patterned gold structure decreased under the tensile stress 


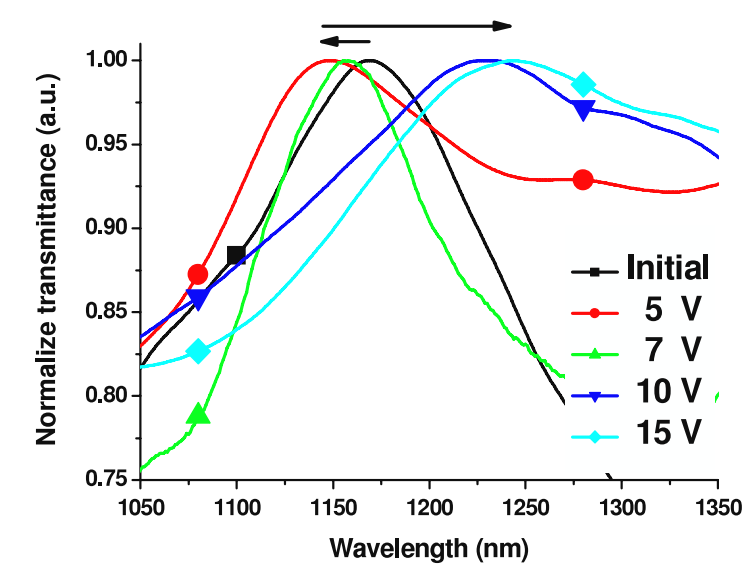

\begin{tabular}{|c|c|c|c|c|c|}
\hline Voltage (V) & 0 & 5 & 7 & 10 & 15 \\
\hline Sites $(\mathrm{nm})$ & 1169 & 1148 & 1158 & 1227 & 1244 \\
\hline$\Delta \lambda(\mathrm{nm})$ & 0 & -21 & -11 & 58 & 75 \\
\hline
\end{tabular}

(a)

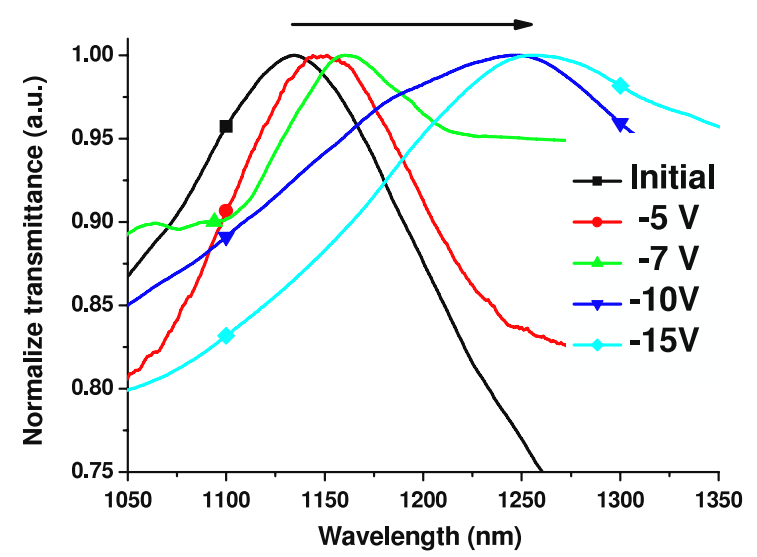

\begin{tabular}{|c|c|c|c|c|c|}
\hline Voltage $(\mathrm{V})$ & 0 & -5 & -7 & -10 & -15 \\
\hline Sites $(\mathrm{nm})$ & 1135 & 1151 & 1164 & 1246 & 1256 \\
\hline$\Delta \lambda(\mathrm{nm})$ & 0 & 16 & 29 & 111 & 121 \\
\hline
\end{tabular}

(b)

Figure 5. Transmittance spectra and SPR wavelength shifts of patterned PZT/gold/PZT structures under different various (a) positive voltage and (b) negative voltage potentials

of the piezoelectric materials, causing the SPR wavelength to blueshift accordingly. Because the refractive index of the surrounding PZT film increased at the same time, which would increase the SP resonance wavelength, the two competing phenomena resulted in the observed experimental results in figure 5(a): an initial blueshift of SPR wavelength under small positive potential with a subsequent redshift of the SPR band upon further increasing the positive potential.

As depicted in figure 4(c), applying a negative potential compressed the patterned PZT/gold/PZT structure; therefore, we expected its period to increase under the compressive stress of the piezoelectric materials. At the same time, the refractive index of the surrounding PZT film increased upon increasing the negative potential. Both of these phenomena lead to shifting of the SPR band to longer wavelength, consistent with the experimental observations in figure 5(b).

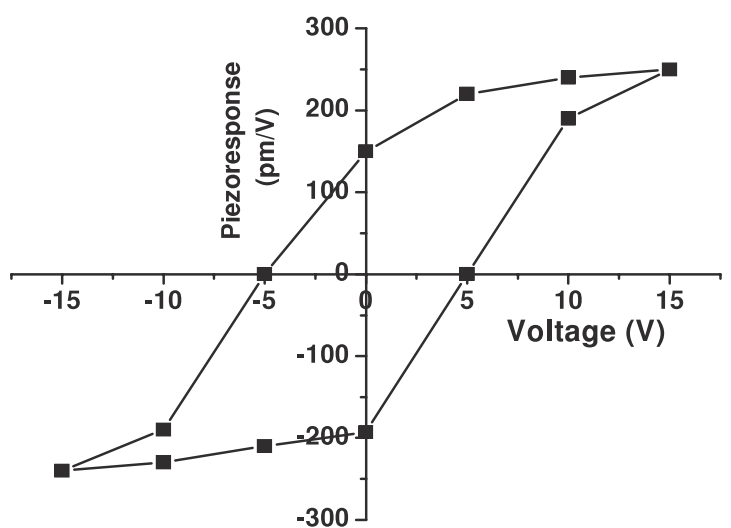

Figure 6. Piezoelectric coefficient $\left(d_{33}\right)$ of PZT films plotted as a function of the applied voltage potential.

As displayed in figure 2(b), there are air voids randomly distributed in the PZT/gold/PZT structure after the sintering process. On applying an external electric field, the PZT could compress the voids for the piezoelectric property. This caused the morphology of PZT/gold/PZT structure to undergo nonuniform deformation. On increasing the external field, the structure distortion would decrease the $Q$ value of the SPR spectrum. In further studies, this phenomenon might be alleviated by the optimization of the sintering process.

To further realize the tunable SPR phenomena of these PZT films, we studied the piezoresponse of the PZT films. An applied field produces a proportional expansive or contractive strain on a PZT film. The proportionality constant, i.e. the piezoelectric coefficient $d$, is numerically identical for both direct and converse effects. Because the piezoelectric effect induces changes in the thickness of a PZT film-either increases or decreases - depending on the sign of the applied electric field, the profiles of the PZT/gold/PZT structures should have expanded or contracted as a result of the piezoelectric response. Figure 6 reveals that the piezoelectric coefficient parallel to the electric field $\left(d_{33}\right)$ depended on the applied voltage. The hysteresis curve of the piezoelectric coefficient of the PZT film reveals an apparent piezoelectric property in the dimensional change. The dimensional change was initially rapid, but then became saturated upon increasing the external potential. In our system, the piezoelectric coefficient perpendicular to the electric field $\left(d_{31}\right)$ had a greater effect on the change in the period of the patterned PZT/gold/PZT structures. Assuming a constant volume for the PZT film, the value of $d_{31}$ is directly proportional to the value of $d_{33}$. Therefore, we can use the piezoelectric curve in figure 6 to estimate the change in period of the patterned PZT/gold/PZT structures.

In this study, under positive applied potential, the decreasing period of the metal structure and the increasing refractive index of the surrounding medium had competing effects on the shift of the SPR wavelength. When applying potentials up to $+5 \mathrm{~V}$, the effect of the decreased period dominated and the SPR peak shifted to shorter wavelengths. Upon increasing the applied potential up to $+15 \mathrm{~V}$, the increased refractive index of the surrounding PZT film 
dominated, redshifting the SPR peak by up to $75 \mathrm{~nm}$ relative to its initial position. These results are consistent with the previous refractive index change and piezoelectric analysis. Upon increasing the positive external potential, the refractive index change of the PZT films was initially small, but it increased dramatically thereafter; in contrast, the dimensional changes in the PZT films were initially rapid, eventually saturating upon increasing the positive external potential. When the PZT/gold/PZT structure was subjected to increasingly negative applied potentials, the increases in both the structural period of the patterned gold and the refractive index of the PZT films both favored redshifting of the SPR peak by as much as $120 \mathrm{~nm}$.

\section{Conclusion}

We have used a direct imprinting method to prepare a bidirectionally tunable SPR filter. A periodic metal/ferroelectric film stack exhibiting SPR phenomena was directly imprinted using a sharp mold in a one-step process. To tune the SPR wavelength, both the refractive index of the surrounding PZT films and the period of the textured PZT/metal/PZT structure were influenced by both the absolute value and sign of the applied potential. Under a positive applied potential, the decreasing structural period and increasing refractive index of surrounding medium had competing effects on the shift in the SPR wavelength, leading to a blueshift of the SPR wavelength at potentials of up to $+5 \mathrm{~V}$ and a subsequent redshift upon increasing the potential further. When applying a negative potential to the patterned PZT/gold/PZT structure, the increases in both the structural period and the refractive index of the PZT films combined to redshift the SPR peak dramatically. As a result, the textured PZT/gold/PZT-based tunable filter modulated the resonance wavelengths over a range of greater than $100 \mathrm{~nm}$ when applying small potentials. The direct imprinting method has great potential for use in the fabrication of tunable optical filters without the need of to employ complicated optical multilayer thin films or liquid crystals. It could also be used for the preparation of other optoelectronic devices exhibiting tunable SPR phenomena.

\section{Acknowledgments}

We thank the National Science Council, Taiwan, Republic of China, for supporting this study under projects NSC-95-2221E-002-324-MY2 and NSC-96-2623-7-002-005-ET.

\section{References}

[1] Christ A, Zentgraf T, Kuhl J, Tikhodeev S G, Gippius N A and Giessen H 2004 Phys. Rev. B 70125113

[2] Bozhevolnyi S I, Erland J, Leosson K, Skovgaard P M W and Hvam J M 2001 Phys. Rev. Lett. 86 3008-11

[3] Ebbesen T W, Lezec H J, Ghaemi H F, Thio T and Wolff P A 1998 Nature $391667-9$

[4] Degiron A, Lezec H J, Barnes W L and Ebbesen T W 2002 Appl. Phys. Lett. 81 4327-9

[5] Barnes W L, Dereux A and Ebbesen T W 2003 Nature 424 824-30

[6] Altewischer E, Exter M P V and Woerdman J P 2002 Nature 418 304-6

[7] Liu W C and Tsai D P 2001 Phys. Rev. B 65155423

[8] Kehagias N, Reboud V, Chansin G, Zelsmann M, Jeppesen C, Schuster C, Kubenz M, Reuther F, Gruetzner G and SotomayorTorres C M 2007 Nanotechnology 18175303

[9] Zhaoning Y et al 2006 Nanotechnology 17 4956-61

[10] Chou S Y, Keimel C and Gu J 2002 Nature 417 835-7

[11] Harnagea C, Alexe M, Schilling J, Choi J, Wehrspohn R B, Hesse D and Gosele U 2003 Appl. Phys. Lett. 83 1827-9

[12] Scott J F, Kammerdimer L, Parris M, Trayner S, Ottenbacher V, Shawabke A and Oliver W F 1988 J. Appl. Phys. 64 787-92

[13] Kang H S and Lee W J 2002 J. Vac. Sci. Technol. A 20 1498-504

[14] Alexe M, Harnagea C, Hesse D and Gosele U 2001 Appl. Phys. Lett. $79242-4$

[15] Buhlmann S, Dwir B, Baborowski J and Muralt P 2002 Appl. Phys. Lett. 80 3195-7

[16] Hsieh K C, Chen H L, Lin C H and Lee C Y 2006 J. Vac. Sci. Technol. B 24 3234-8

[17] Krishnan A, Thio T, Kim T J, Lezec H J, Ebbesen T W, Wolff P A, Pendry J, Martin-Moreno L and Garcia-Vidal F J 2001 Opt. Commun. 200 1-7

[18] Kim T J, Thio T, Ebbesen T W, Grupp D E and Lezec H J 1999 Opt. Lett. $24256-8$

[19] Huang Z, Meng X, Yang P, Zhang Z and Chu J 2000 Appl. Phys. Lett. 76 3980-2

[20] Chen H L, Lee H F, Chao W C, Hsieh C I, Ko F H and Chu T C 2004 J. Vac. Sci. Technol. B 22 3359-62 